\title{
$F$-centers mechanism of long-term relaxation in lead zirconate-titanate based piezoelectric ceramics. 2. After-field relaxation
}

\author{
V. M. Ishchuk* and D. V. Kuzenko \\ Science \& Technology Center "Reaktivelektron" \\ of the National Academy Science of Ukraine \\ Bakinskikh Komissarov Street, 83049 Donetsk, Ukraine \\ *valerii.ishchuk@gmail.com
}

Received 5 May 2016; Revised 12 June 2016; Accepted 6 July 2016; Published 8 August 2016

\begin{abstract}
The paper presents results of experimental study of the dielectric constant relaxation during aging process in $\mathrm{Pb}(\mathrm{Zr}, \mathrm{Ti}) \mathrm{O}_{3}$ based solid solutions (PZT) after action of external DC electric field. The said process is a long-term one and is described by the logarithmic function of time. Reversible and nonreversible relaxation process takes place depending on the field intensity. The relaxation rate depends on the field strength also, and the said dependence has nonlinear and nonmonotonic form, if external field leads to domain disordering. The oxygen vacancies-based model for description of the long-term relaxation processes is suggested. The model takes into account the oxygen vacancies on the sample's surface ends, their conversion into $F^{+}$- and $F^{0}$-centers under external effects and subsequent relaxation of these centers into the simple oxygen vacancies after the action termination. $F$-centers formation leads to the violation of the original sample's electroneutrality, and generate intrinsic DC electric field into the sample. Relaxation of $F$-centers is accompanied by the reduction of the electric field, induced by them, and relaxation of the dielectric constant, as consequent effect.
\end{abstract}

Keywords: Piezoelectric; after-heating process; long-term relaxation; dielectric constant; $F$-center.

\section{Introduction}

Piezoelectric ceramics on the basis of lead titanate-zirconate (PZT) find wide practical applications as sensors, electromechanical devices, transformers and converters, ultrasound sources, etc. ${ }^{1-3}$ At the present years, different kinds of sensors are used under the hardening of worldwide requirements for safe work of devices with moving working part in industry, all kinds of transport. ${ }^{4,5}$ Large part of the said sensors operates under "start-stop" condition. But relaxation properties of materials after the removal of external physical actions have been studied insufficiently till this date ${ }^{6-10}$ Now knowledge about mechanisms of after-effect relaxation processes in ceramic piezoelectric materials is less.

In Refs. 11-13 it was shown that after termination of external effect such as heating (annealing), the dielectric constant of the sample decreases during long time. The relaxation may be as reversible well as irreversible depending on experiment's conditions. In the first case, dielectric constant comes back to initial value that was before external action onto the sample. In the latter case, dielectric constant does not take its initial value. It was proposed as the model for description of long-term after-effect relaxation. This model takes into account the oxygen vacancies on the sample's surface ends, their conversion into $F^{+}$and $F^{0}$-centers and subsequent relaxation of these centers into the simple oxygen vacancies after the actions termination.
The present paper is the extension of Ref. 13 and has the aim to study the external DC electric field influence on the after-effect relaxation of the dielectric constant in PZTbased solid solutions and the verification that is proposed in Ref. 13 of $F$-centers model for the case of the after-field relaxation.

The attention to the after-field relaxation is caused by purely practical reasons also. All technological processes of piezoelectric ceramic elements fabrication comprise its polarization. After the completion of the polarization process, the element operating parameters undergo a change for many days in normal conditions - a natural aging of elements takes place. So-called simulated aging is used in order to shorten the aging period. To carry out simulated aging by the most efficient way, we need to know and understand the mechanism (or mechanisms) of after-effect relaxation.

\section{Brief View of the Model}

In Refs. 11-13, three peculiarities of long-time after-effect relaxation in PZT-based ceramics were emphasized.

(1) Each external effect in itself lasts a few minutes while the after-effect process stretched for tens of hours.

(2) Dielectric constant decreases only during the relaxation process and never rises, i.e., unidirectional change in

This is an Open Access article published by World Scientific Publishing Company. It is distributed under the terms of the Creative Commons Attribution 4.0 (CC-BY) License. Further distribution of this work is permitted, provided the original work is properly cited. 
the dielectric constant takes place after any external effect.

(3) The common logarithmic relaxation law is carried out at the aging of samples after termination of external effects in all cases not depending on the physical nature of the action (heating, DC electric field, uniaxial pressure and some of their combinations). Relaxation rate is only independent on the effect nature, if the latter does not lead to domain reconstruction (it is the case of low effect intensity).

These factors point out the identical physical processes of relaxation that take place after external actions of different nature on samples.

All three of the above-mentioned features of long-term relaxation allowed us to put forward the model of its physical mechanism, ${ }^{13}$ and experimental results obtained at the studying of after-thermal relaxation confirm the said model completely.

In all cases studied in Refs. 11-13, external actions cause a partial depolarization of the sample (now we discussed the case when domain structure destruction does not take place under external actions). As a consequence, a surface electric charge, uncompensated by the polarization field (pyrocharge), is released on the sample's surface ends. At the termination of the external actions, remnant polarization is returned to its original state and the most part of electric charge released earlier is bound by the restored sample polarization.

Together with the above, electric charges (electrons), released on the sample's surfaces under external actions, are captured by positive charged oxygen vacancies. As a result, $F^{+}$- or $F^{0}$-centers are formed. ${ }^{14-17}$ Defects under discussion are concentrated on the sample's surface ends (for polarized sample) and, to a lesser extent, on heterogeneities of sample. ${ }^{14,17-20}$ As an example, grain boundaries can be such heterogeneities. In depolarized sample, the said defects are concentrated on the domain surface ends.

As for the case of the electric field applied in the direction of polarization, in this case, the excess charge is generated by a voltage source. Partly this charge is captured by oxygen vacancies, and after field switching-off all processes are analogous to ones that were considered above.

Excited $F$-centers are separated from the ground-state (net oxygen vacancy) by a potential barrier. ${ }^{15,20,23}$ The potential barrier height is from 0.3 to $1.5 \mathrm{eV}$ for different oxides. ${ }^{15,21-23}$ In oxides with the perovskite crystal structure, this interval is somewhat narrower. After the termination of external effects on the sample, the relaxation of $F^{+}$- and $F^{0}$-centers to the ground state begins. For the above said heights of potential barriers, the time required to restitution of the original state is ranged from several tens to several hundreds of hours. We observed such relaxation times after external effects of different nature on the sample. ${ }^{11-13}$

Before external effects, piezoelectric element was in electrical neutral state. After the $F$-centers creation, the initial neutral state is disturbed - now $F$-centers represent itself as charged defects. Thus, after external influences termination excess charge of $F$-centers (that did not exist before external action) remains on the sample's face ends within a long time. $F$-centers create an electric field, which leads to the change in the dielectric constant (as a result, to a change in the resonant frequency also). This field is directed against the sample's remnant polarization and exists throughout the entire period of time while charged $F$-centers exist. As far as the $F$-centers decay, the induced electric field tends to zero.

This $F$-centers model gives answers to the questions that were pointed at the beginning of the present section and before to our work ${ }^{13}$ had no answers.

Firstly, there is a significant difference in the times of external effects and after-effect relaxation. During external effects, there is a liberation of electrons that are trapped by oxygen vacancies. For electron capture by oxygen vacancies with the $F$-centers formation, the scale of the times is virtually instantaneous in comparison with which we operate in experiments. The subsequent decay of the charge defects is defined by the potential barrier height and occurred for a long time (tens of hours). As consequence, the long-term relaxation is observed. Therefore, $F$-centers model explains large differences of the duration of external effect and the time of relaxation.

Secondly, in all cases after termination of external effects, there is a decrease of sample's permittivity only. Field induced by $F$-centers is directed against sample's remnant polarization and leads to increase of the dielectric constant. As the induced electric field tends to zero, the dielectric constant tends to decrease.

One very important experiment exists that can confirm or refute the mechanism of long-term relaxation that takes into account the creation and decay of $F$-centers.

If all observable after-effects, that we have considered so far, are caused by pyrocharge and $F$-centers formation, then in substances without polarization such effects should not be existing (at least in so far as it occurs in ferroelectrics). To test this conclusion, the samples must be in all aspects identical to those with spontaneous polarization, but they should not have the spontaneous and remnant polarization. For PZT system at small content of titanium, the low-temperature state (at temperatures below the Curie point $T_{C}=230^{\circ} \mathrm{C}$ ) is antiferroelectric one and spontaneous polarization is absent. In all other aspects, these solid solutions are identical to those that are placed into the region of ferroelectric state of the phase diagram: they are obtained in the same technology conditions, they have similar values of the lattice parameters, they have the same microstructure of the sintered ceramic samples and the structure of lattice defects is the same as in ferroelectric solid solutions.

In Ref. 13 the aging (relaxation) dependences of the dielectric constant for solid solutions PZT 98/2 and 52/48 after being subjected to uniaxial compression were studied. For the first of the said solid solution, the ground state is an 
antiferroelectric one, and for the second solid solution such state is a ferroelectric one. For ferroelectric PZT 52/48 solid solution relaxation of dielectric constant takes place after the termination of uniaxial compression (it lasts during some tens hours) and is described by the logarithmic law. For the antiferroelectric PZT 98/2 solid solution, the relaxation of dielectric constant virtually does not exist. This experiment confirms the $F$-centers model to the full.

The present paper represents the results of experiments made for testing the $F$-centers model for long-term relaxation, when DC electric field is taken as external effect.

\section{Experimental Methods}

The disk-shaped piezoelectric ceramic samples of standard size $d=10 \mathrm{~mm}, h=1 \mathrm{~mm}$ were used in the present studies. The elements were obtained from the PZT-based powders. The investigated PZT solid solutions are located in the "composition ( $\mathrm{Zr} / \mathrm{Ti})$ - temperature" diagram of phase states both in the regions of rhombohedral (Rh) and tetragonal $(T)$ distortions of the perovskite crystal lattice as well as in morphotropic region. The latter is characterized by a coexistence of domains or Rh- and $T$-phases in the sample's volume.

Ceramics samples were obtained by the traditional ceramic technology via the two-stage sintering at $850^{\circ} \mathrm{C}$ and $1200^{\circ} \mathrm{C}$. The single phase nature of ceramics and structure change of the samples under the effect of DC electric field were controlled by the X-ray DGM -3 (diffractometer of general mission) using the filtered $\mathrm{Cu} K \alpha$ emission. The residual porosity of samples was not more than $0.2 \%$. Silver electrodes were used for dielectric and piezoelectric measurements. The polarization of ceramic samples was carried out at a temperature $120^{\circ} \mathrm{C}$ in DC electric field with intensity $3 \mathrm{kV} / \mathrm{mm}$ during $1 \mathrm{~h}$ with the subsequent cooling down to room temperature in the field.

As the external effects, which turn out the sample from the state of initial thermodynamic equilibrium, in our experiments were uniaxial mechanical stress and DC electric field which was put to samples in two different directions - along and against to the polarization direction. The time of endurance of each external action was $10 \mathrm{~min}$. The precision of field measurement was $\pm 0.2 \%$. Before the beginning of the each experiment, samples were kept for at least 1 week under normal conditions.

$\mathrm{X}$-ray diffraction patterns were measured in five days after switching-off DC electric field. Before these measurements, silver electrodes were pickled using nitric acid.

Mechanical uniaxial pressure was created with the spring press with precision $\pm 2 \%$.

Temperature dependencies of dielectric constant $\varepsilon$ were carried out in AC electric field with intensity $0.3 \mathrm{~V} / \mathrm{mm}$ and frequency $1 \mathrm{kHz}$. Temperature dependencies of remnant polarization were obtained by integration of electric charge released under heating and thermal depolarization of the samples. Dielectric hysteretic loops $P_{r}(E)$ were observed at the frequency of $5 \times 10^{-3} \mathrm{~Hz}$ with modified Sawyer-Tower circuit.

The aged procedure after each switching off DC field was carried out at room temperature $\left(22^{\circ} \mathrm{C}\right)$ during $30-40 \mathrm{~h}$ and dielectric constant was measured during that time.

\section{Experimental Results}

All processes in ferroelectrics are defined by the behavior of the spontaneous polarization that is the order parameter. But spontaneous polarization does not come under the direct measurements. The most appropriate physical characteristic, that is available for observation and carries information about the order parameter, is the dielectric constant measured in weak fields $(\varepsilon=d P / d E)$. Its measurement at each time takes 1-2 s, so behavior of dielectric constant allows to follow the relaxation processes in the early stages. Another argument for using the dielectric constant in experiments is the large accuracy of its measurement.

For all our experiments, the relaxation of dielectric constant $\varepsilon$ after the termination of external action is described by logarithmic time function

$$
\varepsilon(t) / \varepsilon_{0}=-A \times \ln \left(t / t_{0}\right)+B,
$$

where $t$ is aging time after removal of external action, $\varepsilon(t)$ is the dielectric constant measured at the moment of time $t$ and $t$ is aging time, $\varepsilon(0)$ is the dielectric constant measured before external action. In such entry of the relaxation law, $A$-coefficient corresponds to the relaxation rate. This expression can be rewritten in a form which is adopted in the study of relaxation processes:

$$
\varepsilon(t) / \varepsilon_{0}=-A \times \ln (t / \tau),
$$

where $\tau=\exp (B / A)$. As an example, Fig. 1(a) show the time dependences of dielectric constant after electric field switching-off for PZT-based solid solution (the more detailed discussion of Fig. 1 is shown below). Such relaxation low is fulfilled after termination of sample's heating also. ${ }^{11-13}$

The experimental logarithmic dependence points the wide range of relaxation times. ${ }^{24,25}$ These equations are used at studying of ferroelectric ceramics as well as ferrites.

First of all, let us remind one the main result obtained at the studying of after-heating relaxation. ${ }^{13}$

The dependences of the relaxation rate $A$ (or relaxation time $\tau$ ) on annealing temperature $T_{\text {ann }}$ have nonlinear character and are characterized by the maxima at $T_{\text {ann }}=T_{d}<T_{C}$ for A-coefficient (minimum for $\tau$ ) (see Fig. 5 in Ref. 13). At temperatures $T_{\text {ann }}<T_{d}$ domain reorientations does not occur, and reversible changes of the dielectric constant take place. ${ }^{13}$

For $T_{\text {ann }}>T_{d}$, a destruction of the domain ordering is observed. Partial disordering of the domain structure is confirmed by the variation in the profile of X-ray lines on the 


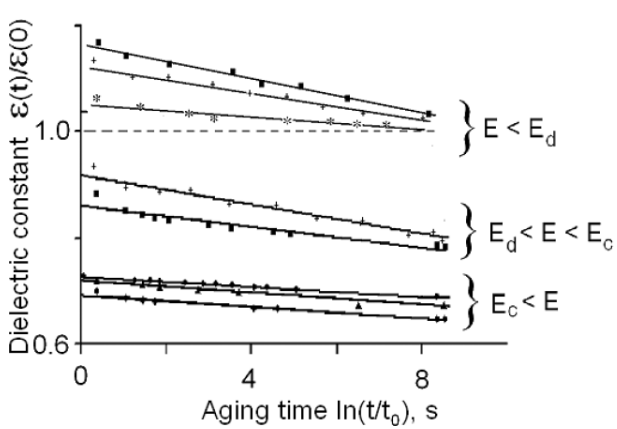

(a)

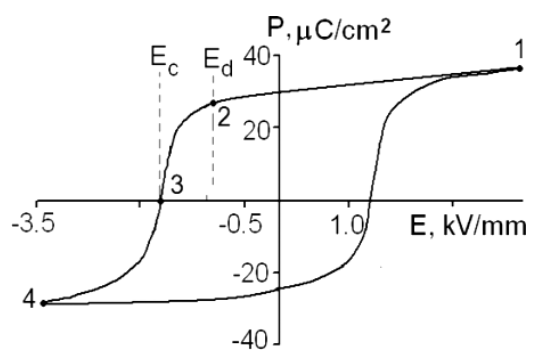

(b)

Fig. 1. (a) - dielectric constant versus aging time ( $\left.t_{0}=1 \mathrm{~min}\right)$ for hard $\left(\mathrm{Pb}_{0,95} \mathrm{Sr}_{0,05}\right)\left(\mathrm{Zr}_{0,53} \mathrm{Ti}_{0,47}\right) \mathrm{O}_{3}$ based ceramics after switching-off DC field with different intensity (field is directed against sample's polarization) (marks of different field intervals in the right from dependences correspond to that showed in Fig. 1(b)). (b) - the loop of dielectric hysteresis, $E_{d}=1.05 \mathrm{kV} / \mathrm{mm}, E_{c}=1.72 \mathrm{kV} / \mathrm{mm}$.

X-ray patterns. ${ }^{13}$ For the samples annealed at the temperature $T_{d}<T_{\text {ann }}<T_{C}$, there is a significant redistribution of the intensity of the doublet lines components, what point to the domain disordering. For the last annealing temperature interval, the change of the dielectric constant has irreversible character.

The explanation of all the above demonstrated experimental result was given on the basis of $F$-centers model. ${ }^{13}$ The increase in the relaxation rate (decrease of the relaxation time $\tau$ ) with increasing annealing temperature in the temperature range $T<T_{d}$ is caused by the fact that an increase in temperature leads to greater decrease of the remnant polarization of the sample and, accordingly, to the greater value of the released charge (electrons).

After sample annealing and rapid cooling $F^{+}$and $F^{0}$-centers are formed. $F^{0}$-center energy is greater than the energy of $F^{+}$-center, so their number on the surface ends of the sample is less and their contribution to the relaxation process is weaker than the contribution of $F^{+}$-centers. When $T_{\text {ann }}$ increases, realized charge increases and the number of $F^{0}$-centers also increases. Thus, their contribution to the electric field, induced by charged $F$-centers increases. Since $F^{0}$-centers are energetically less stable than $F^{+}$-centers, they relax into the oxygen vacancies $\left(F^{0} \rightarrow V_{O}^{*}\right)$ faster. On this reason, induced electric field diminishes with larger rate. Therefore, an increase in annealing temperature (for
$T_{\text {ann }}<T_{d}$ ) results in the increase in the relaxation rate - this is manifested in the increase in the coefficient $A$ and decrease in the relaxation time $\tau$.

When annealing temperatures exceed $T_{d}\left(T_{\text {ann }}>T_{d}\right)$ a partial destruction of domain ordering occurs in polarized sample (there is a turn of the direction of the spontaneous polarization of each domain from the polarization direction of the sample as a whole, and remnant polarization $P_{r}$ decrease as consequence). On this reason, less electric charge is realized on the face ends of the sample during annealing (because the part of realized charge is concentrated on the domain faces that are not parallel to sample's surfaces). Naturally, the amount of charged $F^{0}$-centers is reduced relative on the number of $F^{+}$-centers. All this leads to a decrease of the relaxation rate for the annealing temperatures at $T_{\text {ann }}>T_{d}$.

Now let us pass to experiments with attracting DC electric field.

Electric field does not lead to a change of the nature of $A\left(T_{\text {ann }}\right)$ and $\tau\left(T_{\text {ann }}\right)$ curves. However, the temperature $T_{d}$ depends on the field direction and intensity. When the field and sample's polarization are directed against one to other, dependences $A\left(T_{\text {ann }}\right)$ and $\tau\left(T_{\text {ann }}\right)$ are shifted to lower temperatures. When the field direction coincides with the direction of polarization, these dependences are shifted to the high temperature region. ${ }^{13}$ The dependence of the critical temperature $T_{d}$ on the field intensity $\left|T_{d}\right|(E)$ is practically linear in the range of fields until no partial depolarization of the sample happens (as long as the dependence $P(E)$ is linear in the hysteretic loop - it is the interval $1-2$ in Fig. 1(b)). Such shift of $T_{d}$ corresponds to the change of the stability of domain ordered state in electric field of different orientation.

In other words, the electric field is not a factor that prevents the applicability of the $F$-centers model to the relaxation after-heating process in PZT-based solid solutions.

Further, the results of the study of relaxation processes after an action and switching-off DC electric field rearranging the domain structure are presented. In all cases, the behavior of the dielectric constant after switching-off field is described by the logarithmic relaxation law Eq. (1) (Fig. 1(a)).

We have considered and discussed early in Refs. 11 and 12 the effect of DC field not rearranging the domain structure and after-effect relaxation. After switching-off DC field with sufficiently weak intensity (or any field intensity directed along the polarization of the sample), it is a restoration of the properties of the sample to the initial values.

When DC field is directed opposite to the polarization, there is the critical field $E_{d}\left(E_{d}<E_{c}\right.$, here $E_{c}$ is the coercive field). This field is characterized by two features. Firstly, when $E<E_{d}$, the permittivity relaxes to its initial value (Figs. 1(a) and 2). Secondly, when $E_{d}<E<E_{c}$, dielectric constant during the aging process is not returned to the original value - its final value is less than the initial one. Figure 2 shows the dependences of the dielectric constant on effected electric field obtained after different aging times. Curve 3 in this figure demonstrates the field independence 


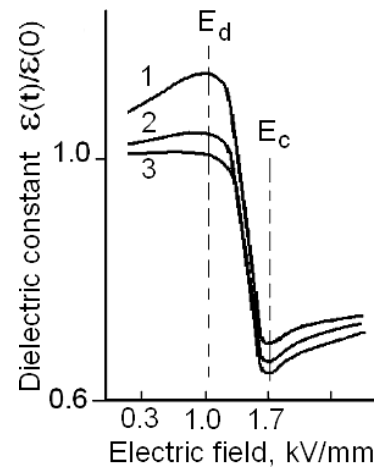

Fig. 2. Relative dielectric constant versus effected electric field obtained after different aging time $\tau$ after the field switching-off (electric field is directed against sample's polarization). $\tau$, h: $1-1 / 6$, $2-5,3-100$.

of this parameter on the field intensity for fields $0<E<E_{d}$ for long term aging $(100 \mathrm{~h})$ and significant dependence for stronger fields. As it can be seen, for $E<E_{d}$ and long relaxation times, the dielectric constant relaxes to its initial value.

Thus, fields $E>E_{d}$ cause nonreversible changes in the sample. The field dependence of the coefficient $A$ (defined by Eq. (1)) goes through a maximum at $E=E_{d}$ (Figs. 3 and 4).

Relaxation processes in polarized and nonpolarized samples have the same character, but differ in their speed - in polarized samples relaxation rate is larger. The field dependence of the coefficient $A$ for unpolarized samples has weaker pronounced maximum in comparison with polarized ones (Fig. 3).

In hard piezoelectric ceramics, the relaxation rate is higher than in soft ones (Fig. 4). Different positions of characteristic temperature $T_{d}$ for soft and hard ceramics are caused by their different coercitive fields.

All these are analogous to that we observed in the study of the after-heating relaxation processes. ${ }^{13}$

$\mathrm{X}$-ray diffraction patterns show that depolarization process in the samples under the effect of the field directed against to the sample's polarization starts earlier than the field reaches the value of the coercive field.

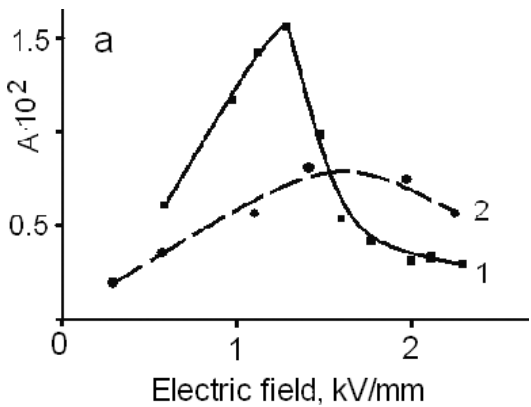

Fig. 3. Field dependence of relaxation rate $(A$-coefficient from Eq. (1)) for polarized (1) and depolarized (2) ceramics of $\left(\mathrm{Pb}_{0,95} \mathrm{Sr}_{0,05}\right)\left(\mathrm{Zr}_{0,53} \mathrm{Ti}_{0,47}\right) \mathrm{O}_{3}$ based solid solution (a). External electric field directed against sample's polarization.

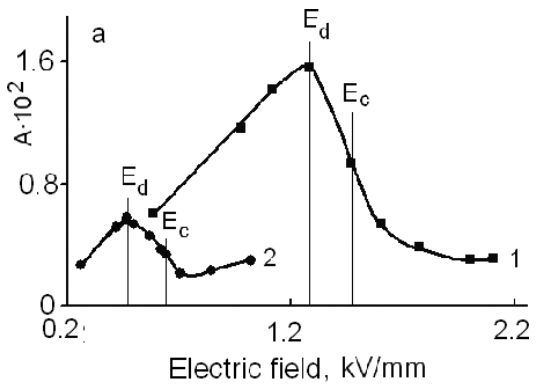

Fig. 4. Field dependence of relaxation rate $(A$-coefficient from Eq. (1) for hard (1) and soft (2) PZT ceramics obtained on the basis of $\left(\mathrm{Pb}_{0.95} \mathrm{Sr}_{0,05}\right)\left(\mathrm{Zr}_{0,51} \mathrm{Ti}_{0,49}\right) \mathrm{O}_{3}$ solid solution (a). External electric field directed against sample's polarization.

Field interval $E_{d}<E<E_{c}$ is characterized by the domains restructuring, which is accompanied by changes in the intensitiy of the doublet components of X-ray lines line (200) for tetragonal solid solutions and lines (220) and (222) for rhombohedral solid solutions (the behavior of these lines allows us to follow for the reconstruction of the domain structure most simply). Field $E_{d}$ is clear threshold electric field for X-ray lines profile change. Until $E<E_{d}$, the form of $\mathrm{X}$-lines is only unchanged. As an example, Fig. 5 shows the change of the pseudo-cubic (200) line for $\left(\mathrm{Pb}_{0,95} \mathrm{Sr}_{0,05}\right)$ $\left(\mathrm{Zr}_{0,51} \mathrm{Ti}_{0,49}\right) \mathrm{O}_{3}$ solid solution (ferro-hard solid solution). This solid solution is located in the tetragonal side of the morphotropic region of PZT phase diagram. Until $E<E_{d}$, intensities of $(002)_{T}$ and $(200)_{T}$ components of X-ray line $(200)_{C}$ are unchanged with field intensity (curves $1-3$ ), but are dependent on the field for $E>E_{d}$ (curves 4-6).

Under electric field directed along the sample's polarization, the change in the character of the profile of X-ray lines is almost absent.

Thus, the field $E_{d}$ (and maximal relaxation rate, respectively) corresponds to the onset of the domain structure

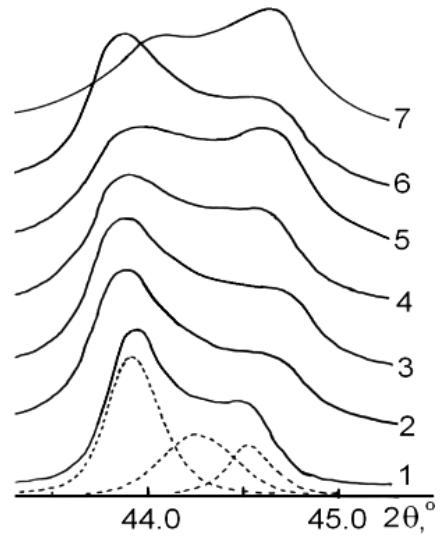

Fig. 5. Change of the shape of the pseudo-cubic (200) line for $\left(\mathrm{Pb}_{0,95} \mathrm{Sr}_{0,05}\right)\left(\mathrm{Zr}_{0,53} \mathrm{Ti}_{0,47}\right) \mathrm{O}_{3}$ solid solution after an action of external DC electric field. $E_{d}=1.05 \mathrm{kV} / \mathrm{mm}, E_{c}=1.72 \mathrm{kV} / \mathrm{mm} . E, \mathrm{kV} / \mathrm{cm}: 1$ and $7-0.00,2-0.62,3-0.91,4-1.11,5-1.54,6-2.53$. 


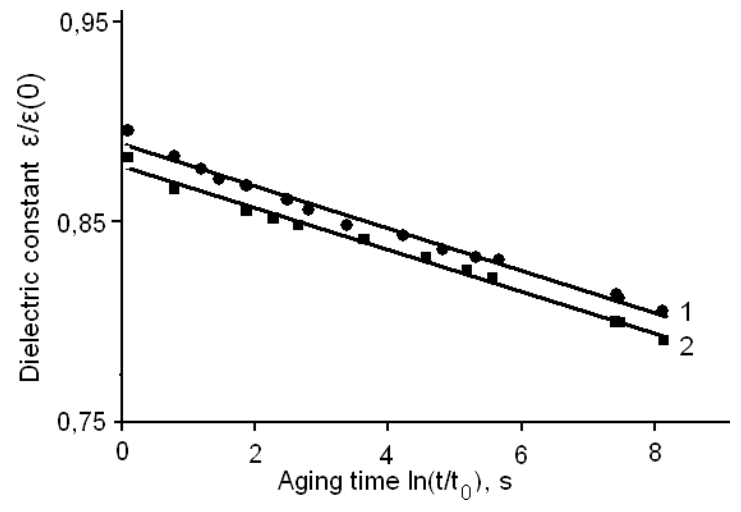

Fig. 6. Time dependence of dielectric constant for hard ceramics of $\left(\mathrm{Pb}_{0,95} \mathrm{Sr}_{0,05}\right)\left(\mathrm{Zr}_{0,53} \mathrm{Ti}_{0,47}\right) \mathrm{O}_{3}$-based solid solution after switching-off DC field $1.6 \mathrm{kV} / \mathrm{mm}$ effected on the sample without (1) and in the combination with uniaxial press $35 \mathrm{MPa}$ (2).

reconstruction. Of course, the major reorganization of the domain structure occurs in the fields close to $E_{c}$.

Additional application of uniaxial compression (up to $200 \mathrm{MPa}$ ) to the sample at the same time with DC electric field does not change the relaxation rate (Fig. 6). Such behavior testifies a more significant influence of electric field on the relaxation characteristics in comparison with uniaxial compression.

This together with the reversibility of dielectric constant only after the pressure action, is the consequence of the fact that used in the paper pressures do not lead (as opposed to electric field) to substantial rearrangement of the domain structure of the samples - uniaxial pressure of about $500 \mathrm{MPa}$ and higher is necessary for the rearrangement of the domain structure.

$\mathrm{X}$-ray patterns were obtained through $120 \mathrm{~h}$ after DC field switching-off. Field was directed against sample's polarization. X-line profiles 1 and 7 correspond to polarized and depolarized sample's state accordingly.

\section{Discussion}

This paper presents the physical effects that accompany the after-field-action relaxation. They can be explained in the framework of the model, proposed earlier in Ref. 13. In this model, the relaxation is caused by the evolution of oxygen lattice vacancies. As in the case of thermal annealing, partly depolarization of the sample under the field action leads to realization of electric charges on the surface ends and creation of $F$-centers. The formation of charged $F^{+}$- or $F^{0}$-centers

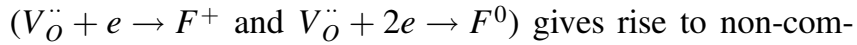
pensated electric field in the sample (directed against sample's polarization) and increasing the dielectric constant. In the process of relaxation of charged vacancies, there is a reduction of the said field and decreases its value.

$F^{0}$-center energy level is above the level of $F^{+}$-center, so their number on the sample surface ends is less and their contribution to the relaxation process is less than the contribution of $F^{+}$-center ones. With an increasing of the charge on the surface ends, the number of $F^{0}$-centers grows and therefore their contribution to the relaxation process increases. Since $F^{0}$-centers energetically is less stable than the $F^{+}$-centers and their stabilization energy is less than corresponding energy of $F^{+}$-centers, so the relaxation into oxygen vacancies $\left(F^{0} \rightarrow V_{O}\right)$ is faster.

At the action of the external field with intensity $E<E_{d}$ (recall, field is directed against the sample's polarization vector) a partial "turn-away" of the polarization vector of domains on the direction of the entire sample's polarization happens without rearrangement of the domain structure. After switching-off the external power supply, the excessive electric charge of $F$-centers remain at the face ends of the sample. The higher the intensity of this field, the greater the accumulated charge at the ends of the sample, greater the relative amount of formed $F^{0}$-centers, higher is the relaxation rate of dielectric constant. The relaxation rate increases with increasing field intensity in the graphs $A(E)$ as Figs. 3 and 4 show.

When the external field strength exceeds $E_{d}$, a partial depolarization of the sample begins via the domains reverse turn. Therefore, after switching-off the field remnant polarization does not return to its initial value, smaller residual charge that came from an external voltage source remains at the end of the sample, smaller amount of $F^{0}$-centers is formed, the relaxation rate decreases. Therefore, in the graphs $A(E)$ in Figs. 3 and 4, the falling segment is observed for fields $E>E_{d}$.

Such character of after-field relaxation and behavior of relaxation rate are similar to ones for the after-heating relaxation. ${ }^{13}$

In partially depolarized samples, less spontaneous polarization domains alignment along the vector of remnant polarization occurs than in polarized one. Less charge is allocated on the sample's surface ends after switching-off external DC field, less charged $F^{+}$- and $F^{0}$-centers are formed on these surfaces. Accordingly, the electric field, which is created by these defects of the crystal lattice, is smaller. Consequently, in depolarized samples, relaxation rate is lower and the curve $A(E)$ lies below (see Fig. 3) than corresponding curve for the polarized sample.

At the study of electric field after-action in the PZT-based solid solutions, similarities and differences intrinsic for hard or soft solid solutions was discovered, for polarized or unpolarized samples (Figs. 3 and 4). The nature of these effects is similar to the nature of the effects that were occurred after samples heating and were discussed in Ref. 13.

The difference of relaxation rate in hard and soft solid solutions (see Fig. 4) finds its explanation in the framework of the $F^{0}$-centred model also. Hard or soft ferroelectric ceramics may be obtained on the basis of the same PZT solid solution, at the same ratio of $\mathrm{Zr} / \mathrm{Ti}$. In the former case, solid solution is doped by monovalent ions, substituted lead in the 
$A$-sublattice of the crystal structure, or trivalent ions substituted $B$-ions of parent solid solution. ${ }^{1}$ Soft PZT based ceramics are obtained in the way of the substitution of lead by trivalent ions or $B$-ions by pentavalent ions. ${ }^{1}$ As a result of compliance with the rule of electrical neutrality, hard ferroelectric ceramics contain more oxygen vacancies. ${ }^{1}$ In the study of the present paper, the piezoelectric soft solid solutions content, the trivalent bismuth as doped element substituted the lead, and ferroelectric hard ceramics is obtained via introducing the trivalent ions (manganese of iron) into $B$-sites of the crystal lattice.

After field action, the above discussed mechanism of electron capture by oxygen vacancies takes effect and $F^{+}$and $F^{0}$-centers are formed. The subsequent relaxation of dielectric constant after switching-off external field is a consequence of the relaxation of these centers to the ground state (to the "pure" oxygen vacancy) and depression to zero the field formed by the said charged defects. More oxygen vacancies in hard ferroelectric ceramics provide larger amount of $F^{0}$-centers, and as a result, larger relaxation rate in the aging process.

\section{Conclusions}

This paper presents the results of the study of relaxation processes in piezoelectric PZT-based solid solutions after external electric field affecting the sample. As a parameter, which we monitor during the experiments, has been selected as dielectric constant. It is selected as most closely associated with the order parameter of ferroelectric ordering.

After the effect of weak electric field directed against the sample's polarization that do not lead to a rearrangement of the domain structure (or field any intensity directed along the sample's polarization), dielectric constant is returned to its initial value (which was before the external action on the sample) during the long-term aging. The dependence of the relaxation rate on the action intensity has linear character.

After DC electric field treatment leading to a rearrangement (full or partial) of the domain structure, relaxation is irreversible - after field action termination the dielectric constant does not return to its initial value. In this case, the dependence of the relaxation rate on the electric field intensity is nonlinear - there is a critical value $E_{d}$ of effected field at which the relaxation rate has maximum. In the vicinity of the said critical field, the domain structure reorientation onsets.

All experimental results stand in the frame of the so-called $F$-centers model. The model takes into account the presence of crystal lattice defects, among which oxygen vacancies are more crucial. Both the remnant polarization decrease and free charge (unbound by polarization) realization at the surface ends of the sample (or domains) take place during the external actions. This charge is captured by oxygen vacancies, and $F^{+}$- and $F^{0}$-centers are formed. After the termination of external effects, $F$-centers induce an electric field inside the sample that results in deviation of dielectric constant from the initial value.

This field is retained after removal of the external influences on the sample during the lifetime of the charged $F$-centers. At the $F$-centers decay $\left(F^{0}, F^{+} \rightarrow V_{O}\right)$, electric field created by them decreases to zero. During the $F$-centers decay, dielectric constant decreases to its initial value also.

The results presented in this publication are caused by the restructuring of the domain structure. However, domain restructuring does not determine the mechanism of aftereffect long-term relaxation. Rearrangement of the domain structure determines peculiarities of relaxation process only, in particular, the nonlinear dependence of the relaxation rate on the annealing temperature, but not own relaxation mechanism.

\section{References}

${ }^{1}$ B. Jaffe, W. R. Cook and H. Jaffe, Piezoelectric Ceramics (Academic Press, London and New-York, 1971).

${ }^{2}$ C. Burfoot and G. W. Taylor, Polar Dielectrics and Their Applications (The McMillan Press, 1979).

${ }^{3}$ Y. Xu, Ferroelectric Materials and Their Applications (NorthHolland, Amsterdam, 1991).

${ }^{4}$ J. Yang, Analysis of Piezoelectric Devices (University of Nebraska-Lincoln, USA, 2006).

${ }^{5}$ C.-C. Wu, C.-C. Lee, G. Z. Cao and I. Y. Shen, Effects of corner frequency on bandwidth and resonance amplitude in designing PZT thin-film actuators, Sens. Actuators A 125, 178 (2006).

${ }^{6} \mathrm{C}$. S. Ganpule et al., Polarization relaxation kinetics and $180^{\circ}$ domain wall dynamics in ferroelectric thin films, Phys. Rev. B $\mathbf{6 5}$, 014101 (2001).

${ }^{7}$ D. Zhoua and M. Kamlah, Determination of room-temperature creep of soft lead zirconate titanate piezoceramics under static electric fields, J. Appl. Phys. 98, 104107 (2005).

${ }^{8}$ E. M. Bourim et al., Creep behavior of undoped and $\mathrm{La}-\mathrm{Nb}$ codoped PZT based micro-piezoactuators for micro-optical modulator applications, Sens. Actuators A 155, 290 (2009).

${ }^{9}$ S.-J. Kima, J. H. Kima and C.-H. Lee, Domain switching and creep behavior of a poled PZT wafer under through-thickness electric fields at high temperatures, Acta Mater. 58, 2237 (2010).

${ }^{10}$ S. A. Gridnev, V. M. Popov and L. A. Shuvalov, The processes of slow relaxation in TGS monocrystals, Izv. Akad. Nauk. SSSR, Ser. Fiz. 48, 1226 (1984) (in Russian).

${ }^{11}$ D. V. Kuzenko, V. M. Ishchuk, A. I. Bazhin and N. A. Spiridonov. Long-time aftereffects and relaxation in piezoelectric ceramics. 1. Linear reversible processes, Ferroelectrics 413, 29 (2011).

${ }^{12}$ D. V. Kuzenko, V. M. Ishchuk, A. I. Bazhin and N. A. Spiridonov, After-effect relaxation process in lead zirconatr-titanate, Fiz. Tverd. Tela 54, 896 (2012) (in Russian).

${ }^{13}$ V. M. Ishchuk and D. V. Kuzenko, $F$-centers mechanism of longterm relaxation in lead zirconate-titanate based piezoelectric ceramics. 1. After-heating relaxation, J. Adv. Dielect. 5, 1550036 (2015).

${ }^{14}$ L. Hong, A. K. Soh, Q. G. Du and J. Y. Li, Interaction of O-vacancies and two-dimensional ferroelectric model, Phys. Rev. B 77, 094104 (2008). 
${ }^{15}$ C. H. Park and D. J. Chadi, Microscopic study of oxygen-vacancy defects in ferroelectric perovskites, Phys. Rev. B 57, R13961 (1998).

${ }^{16}$ A. Stashans, S. Serrano and P. Medina, A quantum-chemical study of oxygen-vacancy defects in $\mathrm{PbTiO}_{3}$ crystals, Physica B 381, 82 (2006).

${ }^{17}$ E. Cockayne and B. P. Burton, Dipole moment of a $\mathrm{Pb}-\mathrm{O}$ vacancy pair in $\mathrm{PbTiO}_{3}$, Phys. Rev. B 69, 144116 (2004).

${ }^{18}$ W. J. Merz, Domain formation and domain wall motions in ferroelectric $\mathrm{BaTiO}_{3}$ single crytals, Phys. Rev. 95, 690 (1954).

${ }^{19} \mathrm{~K}$. Amanuma, T. Hase and Y. Miyasaka, Fatigue characteristics of Sol-Gel derived $\mathrm{Pb}(\mathrm{Zr}, \mathrm{Ti}) \mathrm{O}_{3}$ thin films, Jpn. J. Appl. Phys. Part 1 33, 5211 (1994).

${ }^{20}$ W. H. Shepherd, Fatigure and aging in sol-gel derived PZT thin films, in Ferroelectric Thin Films, ed. E. R. Myers and A. I. Kingon, Materials Research Society Symposium Proceedings No. 200 (Materials Research Society, Pittsburgh, 1990), p. 277.
${ }^{21}$ E. A. Kotomin, R. I. Eglitis and G. Borstel, Quantum chemical modelling of point defects in $\mathrm{KNbO}_{3}$ perovskite crystals, Comput. Mater. Sci. 17, 290 (2000).

${ }^{22} \mathrm{C}$. Duque and A. Stashans, Structural and optical properties of $F$-center defects on barium titanate $<001>$ surface, Mater. Lett. 57, 3954 (2003).

${ }^{23}$ R. I. Eglitis, $A b$ initio calculations of $\mathrm{SrTiO}_{3}, \mathrm{BaTiO}_{3}, \mathrm{PbTiO}_{3}$, $\mathrm{CaTiO}_{3}, \mathrm{SrZrO}_{3}, \mathrm{PbZrO}_{3}$ and $\mathrm{BaZrO}_{3}$ (001), (011) and (111) surfaces as well as $F$-centers, polarons, KTN solid solutions and Nb impurities therein, Int. J. Mod. Phys. B 28, 1430009 (2014).

${ }^{24}$ R. Street, J. C. Woolley and P. B. Smith, Magnetic viscosity under discontinuously and continuously variable field conditions, Proc. Phys. Soc. B 65, 679 (1952).

${ }^{25}$ S. Krupička, Physik der Ferrite (Academia, Prague, 1973) (in German). 